\title{
Vibrationally promoted emission of electrons from low work function surfaces: Oxygen and Cs surface coverage dependence
}

\author{
Jason D. White, Jun Chen, and Daniel Matsiev \\ Department of Chemistry and Biochemistry, University of California, Santa Barbara, \\ California 93106-9510 \\ Daniel J. Auerbach \\ Hitachi Global Storage Technologies, 650 Harry Road, San Jose, California 95120-6099 \\ Alec M. Wodtke ${ }^{\text {a) }}$ \\ Department of Chemistry and Biochemistry, University of California, Santa Barbara, \\ California 93106-9510
}

(Received 4 October 2004; accepted 20 December 2004; published 28 June 2005)

\begin{abstract}
We observe electron emission from low work function Cs covered Au surfaces due to the scattering of vibrationally excited NO molecules with 18 quanta of vibration in the ground electronic state. Additional experiments explore the influence of oxygen exposure on the electron emission. These results indicate a nondissociative mechanism for reactivity of vibrationally excited $\mathrm{NO}$ on these surfaces and provide evidence against an Auger-like mechanism. We note a remarkable similarity between trends of the vibrationally produced electron emission as the surface composition changes and reports on surface work function. (C) 2005 American Vacuum Society.
\end{abstract}

[DOI: $10.1116 / 1.1861942]$

\section{INTRODUCTION}

There is a large amount of current interest in the role of nonadiabatic electronic effects in vibrational energy transfer and chemical reactions at metal surfaces. Generally it is assumed that the Born-Oppenheimer approximation ${ }^{1}$ is valid and that reactions proceed without nonadiabatic changes in electronic state as the electronic rearrangement takes place on a time scale roughly three orders of magnitude slower than that for nuclear motion. In gas phase molecular collisions, the separation between ground and excited electronic states is often sufficiently large and the motion sufficiently slow that reactive collisions are electronically adiabatic. The conditions that insure adiabatic behavior are given by the Massey Criterion

$$
\frac{h \mu \dot{R} \mathbf{d}_{12}}{\left|E_{1}-E_{2}\right|} \ll 1,
$$

where $\mathbf{d}_{12}$ is the nonadiabatic coupling, $\mu$ is the reduced mass, and $\dot{R}$ is the nuclear velocity. Because of the larger number and density of electronic states, when molecules approach a metal surface, the separation between ground and excited states is small and the Massey Criterion can no longer be used to guarantee electronically adiabatic behavior.

Some of the most dramatic effects of vibrationally mediated energy transfer were seen in the collisions of highly vibrationally excited molecules with surfaces ${ }^{2}$ where NO $v$ $=15$ inelastically loses up to 10 quanta of vibrational energy upon scattering from $\mathrm{Au}(111)$. Theoretical work by $\mathrm{Li}$ and Guo $^{3}$ successfully reproduced these results using a mecha-

a) Author to whom correspondence should be addressed; electronic mail: wodtke@chem.ucsb.edu nism that involved nonadiabatic electron transfer events between the neutral and anionic potential energy surfaces of the NO molecule.

Direct observation of such electron transfer events would therefore lend credence to the development of newer models for gas-surface scattering. Clean gold surfaces have a work function on the order of $5.3 \mathrm{eV}^{4}$ and $\mathrm{NO}$ molecules in $v$ $=18$ have vibrational energy of $3.76 \mathrm{eV}$. Clearly, electron emission from vibrational energy transfer events would be unobservable in this system. By reducing the metals work function, this situation changes. Alkali metals have work functions on the order of $1.9-2.5 \mathrm{eV}^{4}$ and a small coverage of an alkali metal on a transition metal produces a surface with an even lower work function ${ }^{5-8}$ which is significantly lower than the vibrational energy of NO in $v=18$.

Exoelectron emission for ground state molecules has been seen for many alkali covered metal systems. A comprehensive survey of these systems can be found in Greber ${ }^{9}$ and references therein. For the widely studied $\mathrm{O}_{2}$ with $\mathrm{Cs}$ films ${ }^{10-12}$ the delayed onset of exoelectron emission with $\mathrm{O}_{2}$ coverage was key to establishing the importance of an Auger mechanism in the emission event.

We have previously reported experiments showing electron emission when $\mathrm{NO}(v=18)$ collides with a $\mathrm{Au}(111)$ surface with a submonolayer coverage of cesium. ${ }^{13}$ In the current article we concentrate on the influence of $\mathrm{Cs}$ and $\mathrm{O}$ coverage on the electron emission probability. This article reports measurements which combine the ability to create highly vibrationally excited $\mathrm{NO}$ molecules and the ability to create a low work function $\mathrm{Cs} / \mathrm{Au}$ surface to observe electrons ejected from the surface upon scattering of NO molecules. Our work, in contrast to previously studied alkali systems, shows electron emission prior to any introduction of $\mathrm{O}_{2}$, which is suggestive of different mechanism than that of 


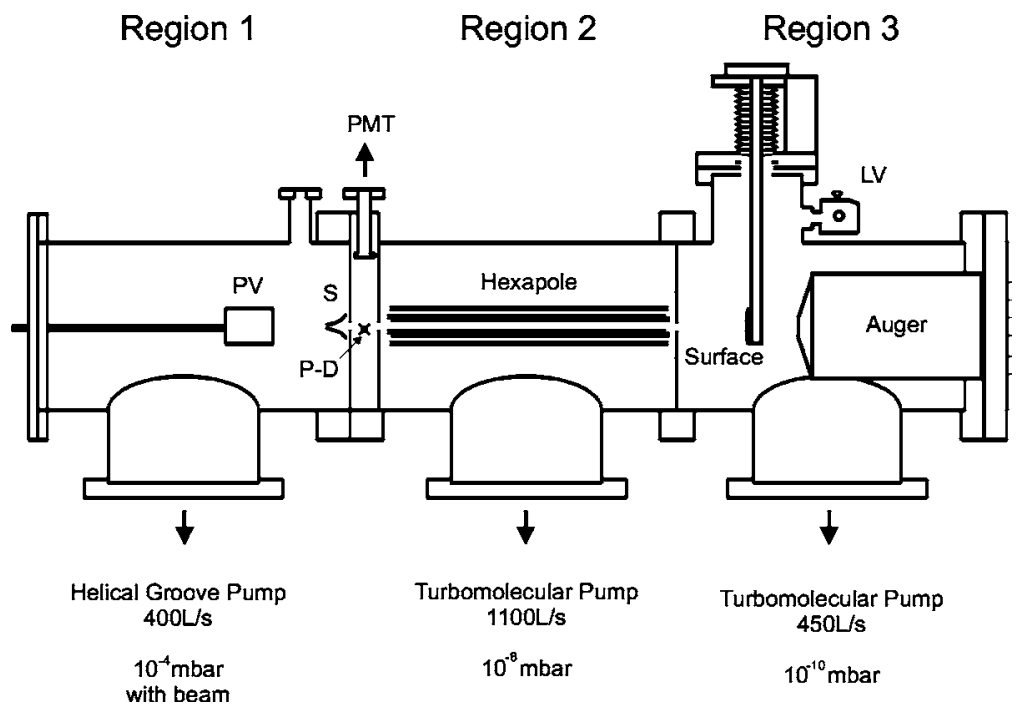

FIG. 1. Experimental apparatus. The supersonic NO molecular beam expands from the pulsed valve (PV) into a region of high vacuum $\left(10^{-7} \mathrm{mbar}\right)$ and is collimated by a electro-formed skimmer (S). Two laseroptical pumping (P-D) is performed after the skimmer and the fluorescence emission and fluorescence depletion are collected with a photomultiplier tube (PMT). The state selectively prepared molecular beam travels downstream through Region 2, which is equipped with an electrostatic hexapole for molecular focusing. The third and final chamber is an UHV $\left(10^{-10} \mathrm{mbar}\right)$ chamber where electron emission is measured and where low work function cesium/gold surfaces are prepared. A UHV leak valve for oxygen gas is mounted onto the manipulator in order to introduce controlled exposures of oxygen gas for oxidation experiments. an Auger process. The dependence of vibrationally promoted electron emission on $\mathrm{Cs}$ and $\mathrm{O}$ coverage is similar to that of the surface's work function.

\section{EXPERIMENT}

The experimental setup is shown schematically in Fig. 1. A molecular beam traveled through three regions of differential pumping, with the final chamber kept at ultrahigh vacuum for low background surface scattering experiments.

Region 1, with base pressure of $10^{-6} \mathrm{mbar}$, had a homebuilt pulsed piezoelectric valve (PV) operating at $10 \mathrm{~Hz}$ which allowed for a supersonic expansion of our gas. A gas mixture of $15 \% \mathrm{NO}$ in $\mathrm{Kr}$ at 3 bar stagnation pressure was expanded from the nozzle to create a molecular beam of rotationally cold $(5 \mathrm{~K}) \mathrm{NO}$ molecules. The beam passed through a $2 \mathrm{~mm}$ collimating electro-formed skimmer (Beam Dynamics, Inc.) into Region 2 where it was optically pumped to NO $v=18$ using stimulated emission pumping (SEP). ${ }^{14,15}$

Commonly referred to as PUMP-DUMP Spectroscopy, SEP uses two lasers tuned to different wavelength and is used to transfer population from the ground state of a molecule through an intermediate electronically excited state to a vibrationally excited level in the ground electronic state. For NO SEP, light around $195.5 \mathrm{~nm}$ was used to excite NO from $X^{2} \Pi_{1 / 2}, v=0$ to $A^{2} \Sigma^{+}, v=3$ via either the $Q_{11}$ or the $R_{11} Q_{21}$ rotational transitions (PUMP). A second laser, tuned to $\sim 460 \mathrm{~nm}$, stimulated emission down to the $A^{2} \Sigma^{+}, v=3$ $\rightarrow X^{2} \Pi, v=18$ (DUMP).

Two separate Nd:yttrium-aluminum-garnet (YAG) pumped dye laser systems were used to generate light for the PUMP and DUMP steps. Tunable light $(\sim 195.5 \mathrm{~nm})$ for the PUMP step was generated by frequency summing in the following manner. An injection seeded Nd:YAG laser (Spectra Physics LAB-170) simultaneously produced $150 \mathrm{~mJ} /$ pulse at $532 \mathrm{~nm}$ and $100 \mathrm{~mJ} /$ pulse at $266 \mathrm{~nm}$. A dye laser (Sirah CSTR-DA-24) operating on LDS 751 was pumped by the $532 \mathrm{~nm}$ light. The tunable output of the dye and $10 \mathrm{~mJ}$ of the
$266 \mathrm{~nm}$ output were frequency summed using a BBO crystal to yield approximately $1 \mathrm{~mJ} /$ pulse near $195 \mathrm{~nm}$. A second dye laser operating at $\sim 460 \mathrm{~nm}$ stimulated emission back to $v=18$. Here, the third harmonic of a Nd:YAG laser (Spectra Physics PRO-200) pumped a dye laser (Sirah PRSC-DA-24) operating on Coumarin 460, which generated $60 \mathrm{~mJ} /$ pulse in the $460-465 \mathrm{~nm}$ region. The counter propagating laser beams were introduced to the chamber through UV viewports and overlapped in space and time with the molecular beam.

Fluorescence resulting from spectroscopic transitions was collected through an imaging lens by a photomultiplier tube (PMT) (Hammamatsu R212UH) mounted at a right angle to the molecular beam and operated at 200-300 V. The signal was monitored and acquired with a $500 \mathrm{MHz}$ LeCroy Waverunner oscilloscope (LT344) interfaced to a PC.

From the preparation region, the vibrationally excited molecular packet traveled through Region 2. Although not used in the current work, the hexapole can be used to enhance the number of vibrationally excited molecules in Region 3 by molecular focusing. Further details can be found in Chen et al. ${ }^{16}$ Once passing through the second chamber, the molecules enter the surface chamber, Region 3, through a 1-mm-diam aperture.

Region 3 , with $4 \times 10^{-10}$ mbar background pressure, contained a room temperature gold crystal ${ }^{17}$ mounted on a translatable and rotatable manipulator. The chamber also contained an Auger spectrometer (Phi Electronics), an argon-ion sputtering gun (Thermo Electronics Corporation-not shown), a quadrupole mass spectrometer (Balzers, not shown), an UHV leak valve (LV) to introduce oxygen gas, and low work function surface preparation equipment as shown in Fig. 2.

Low work function surfaces were prepared in the following manner. The Au surface was sputtered with the Ar ion gun until the surface was cleaned of $\mathrm{O}$ and residual $\mathrm{Cs}$ contaminants as determined by Auger spectroscopy. The surface was then turned toward the SAES [SAES Getters Group (Milan, Italy)] Cs doser (Cs), and a current of 6 A was ap- 


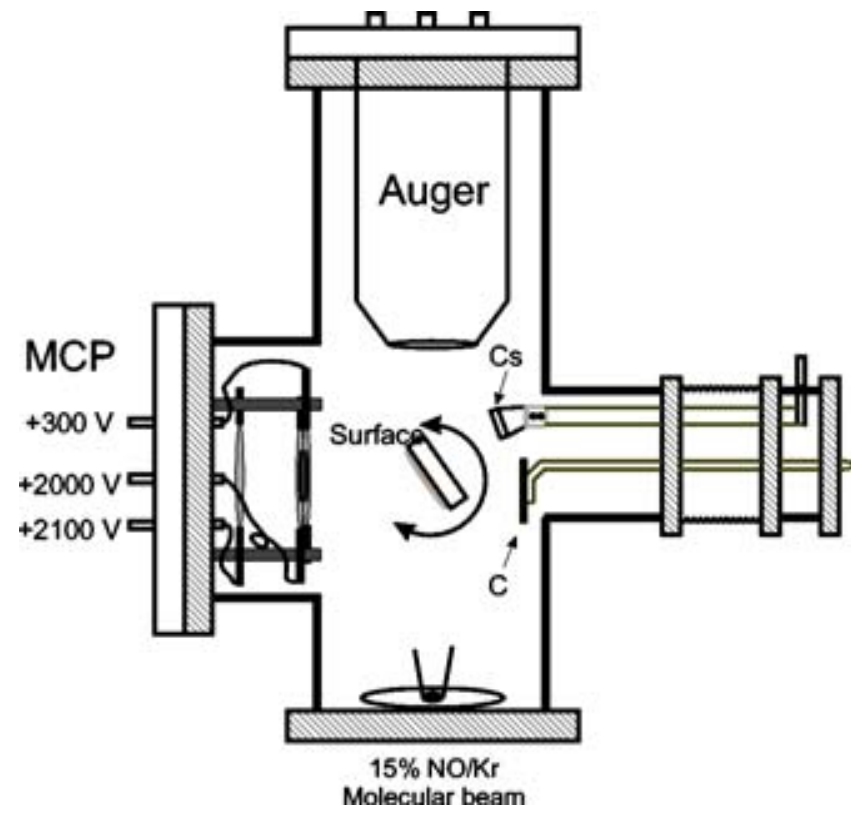

FIG. 2. Detailed top-down view of the UHV surface chamber. For preparation of low work function surfaces, a SAES cesium doser $(\mathrm{Cs})$ is heated (6 A for approximately $30 \mathrm{~s}$ ) to deposit cesium atoms on the gold surface. During deposition, a helium-neon laser is directed through a viewport (not shown) at the surface and emitted photoelectrons are collected with a metal flag $(\mathrm{C})$ biased at $+50 \mathrm{~V}$. The surface can then be rotated $65^{\circ}$ relative to the NO molecular beam towards the multichannel plate detector which is biased as shown to collect the electrons emitted upon scattering of the vibrationally excited NO. The chamber is also equipped with an $\mathrm{Ar}^{+}$sputtering gun (not shown) and Auger spectroscopy to ensure cleanliness of the surface.

plied to the doser to induce Cs atom sublimation. During dosing, a $5 \mathrm{~mW}$ helium-neon (He-Ne) laser operating at $632.8 \mathrm{~nm}(1.95 \mathrm{eV})$ was directed upon the surface to induce photoemission which changes in intensity as a function of the coverage of cesium. In this manner, we were able to prepare surfaces that had the same cesium coverage as well as the maximum in photoemission. It is quite difficult, however, to exactly quantify the amount of Cs on the Au surface. We estimate that the cesium coverage is $0.5 \mathrm{ML}$ at the maximum in photoemission, as per Skottke-Klein. ${ }^{18}$ This is also consistent with our Auger analysis.

A custom built microchannel plate (MCP) detector, constructed of two $40 \mathrm{~mm}$ plates in a chevron configuration, was used to collect negative particles emitted from the surface. The assembly could be set to a limit of the voltages shown in Fig. 2. The increasing positive bias was used to ensure the measurement of negatively charged particles. The MCPs were approximately $5 \mathrm{~cm}$ from the surface during experiments. Figure 2 also shows the orientation of the surface during scattering of NO beam at an angle of incidence of approximately $65^{\circ}$ to the beam normal.

A permanent magnet, capable of producing a 2-3 G magnet field, was used to insure that the resulting signal was from electrons and not negatively charged ions. When the magnet was positioned outside the machine, the total suppression of the signal indicated that the negatively charged particles were indeed due to electrons.

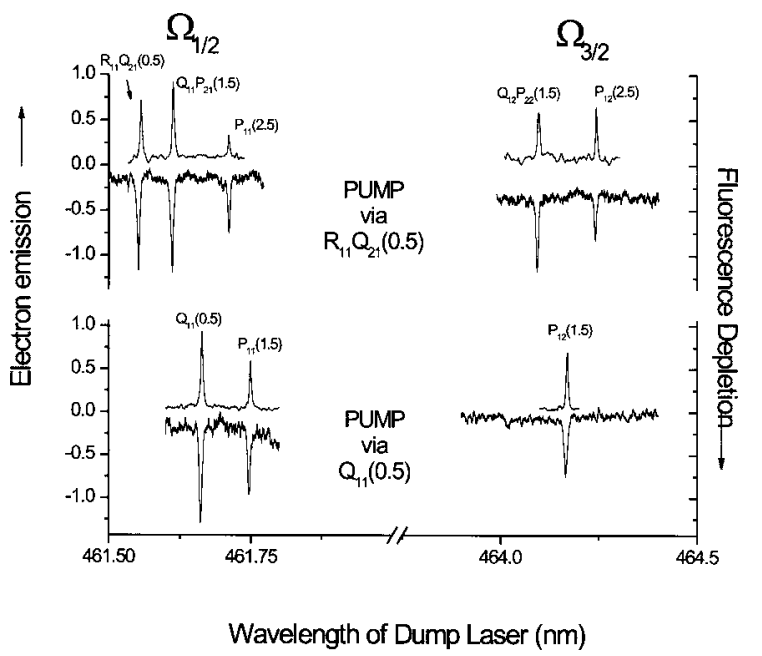

FIG. 3. Dump laser scans at fixed PUMP wavelength. DUMP laser scans are measured in two fashions. Downward-going lines generated by fluorescence depletion measurements. Upward-going lines are detected by the interaction of vibrationally excited $\mathrm{NO}$ with a $\mathrm{Cs} / \mathrm{Au}$ surface. Upper traces utilize $A^{2} \Sigma^{+}, v=3 \leftarrow X^{2} \Pi_{1 / 2}, v=0 R_{11} Q_{21}(J=0.5)$ pump line and the lower traces are from $A^{2} \Sigma^{+}, v=3 \leftarrow X^{2} \Pi_{1 / 2}, v=0 Q_{11}(J=0.5)$. Left-most scan regions populate $X^{2} \Pi_{1 / 2}, v=18 \Omega=0.5$ in the labeled rotational states. The rightmost scans are for $X^{2} \Pi_{1 / 2}, v=18 \Omega=1.5$.

It was interesting to observe the influence of work function on the negative particle emission when vibrationally excited NO was scattered from the surface. One way to accomplish this is by exposure of oxygen to the Cs/Au surface. ${ }^{5,6}$ An UHV leak valve allows for the introduction of $\mathrm{O}_{2}$ gas at a constant pressure of approximately $4^{*} 10^{-9}$ mbar. The particle emission was monitored as a function of oxygen exposure and compared to the photoemission from a He-Ne laser also as a function of oxygen exposure.

\section{RESULTS}

\section{A. Electron emission spectroscopy}

The observed electron emission resulting from the interaction of vibrationally exited $\mathrm{NO}(v=18)$ and the Cs/Au surface is shown as the upward going lines in Fig. 3. These lines are plotted as a function of the DUMP laser wavelength and shown with the correlating fluorescence depletion ${ }^{15}$ spectra (downward going lines). Two PUMP transitions are utilized, $R_{11} Q_{21}(0.5)$ [top part of Fig. 3] and $Q_{11}(0.5)$ [lower part of Fig. 3] to prepare the initial electronically excited state. The labeled ro-vibronic DUMP transitions observed in Fig. 3 can be identified with allowed transitions for a ${ }^{2} \Sigma^{+}-{ }^{2} \Pi$ system. See Fig. 4. It is important to notice that the transitions occur at the predicted transition frequencies ${ }^{19}$ and with the same linewidths $\left[\sim 0.3 \mathrm{~cm}^{-1}\right.$ full width at half maximum (FWHM)] in both spectra. From Fig. 3, it is clear that the detected electron emission signal is directly due to the vibrationally excited NO molecules. 


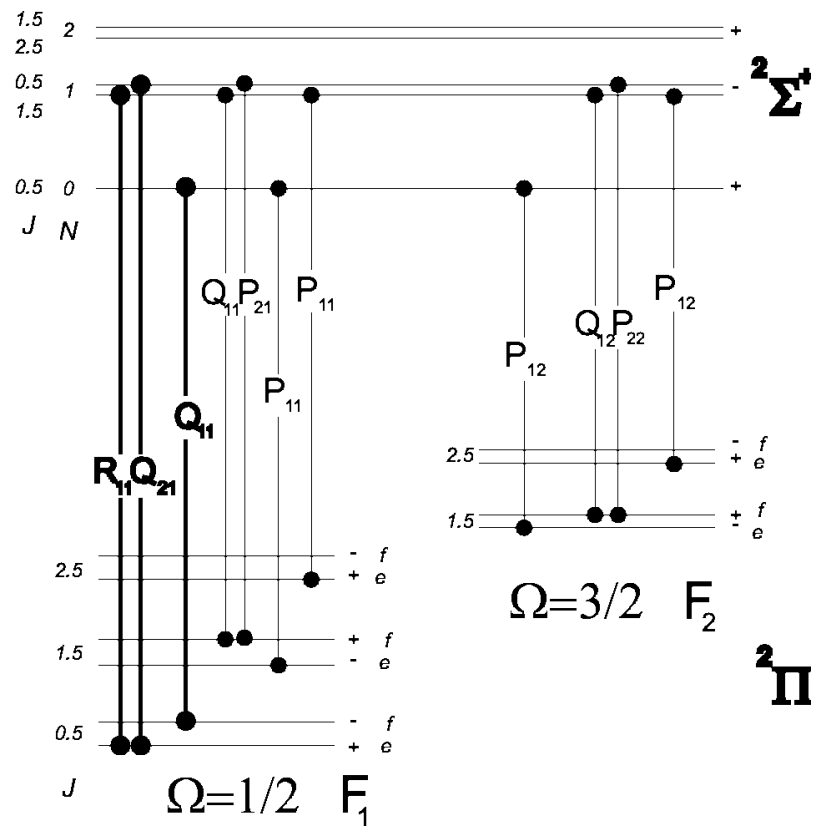

FIG. 4. Selection rules for NO. Allowed transitions for various rotational states of NO from the ${ }^{2} \Pi$ ground state to the ${ }^{2} \Sigma^{+}$excited $A$ state. PUMP lines discussed in Fig. 3 are shown in bold.

\section{B. Results of varying $\mathrm{Cs}$ and $\mathrm{O}_{2}$ exposure}

In this work we have attempted to gain control over the surface work function by means of chemical modification to the surface. Specifically, we have varied the time of exposure for the Au surface to both $\mathrm{Cs}$ and $\mathrm{O}_{2}$.

The vibrationally promoted electron emission resulting from collisions with NO in $v=18$ is shown in Fig. 5 as a

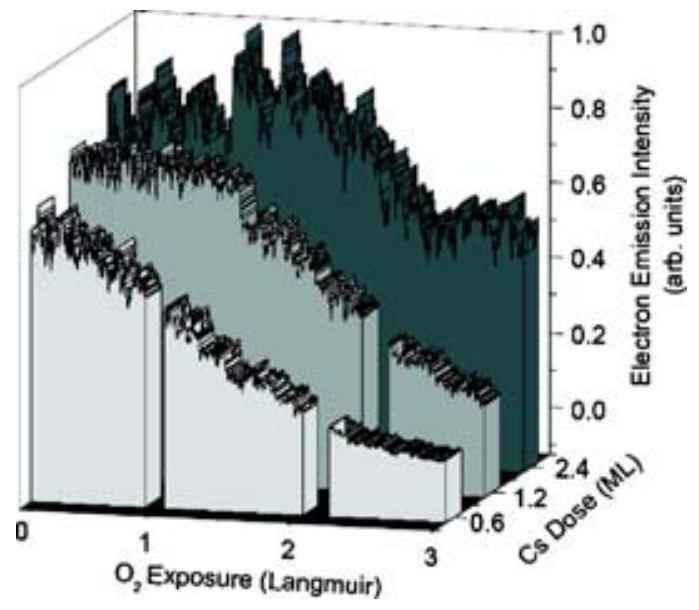

FIG. 5. Electron emission as a function of oxygen exposure. Electron emission due to collisions of $\mathrm{NO}(v=18)$ on low work function surfaces. Emission intensity is monitored vs oxygen exposure for three surfaces with differing initial Cs coverage. Two features of the data stand out. First, electron emission is observed in the limit of zero-oxygen coverage. This stands in stark contrast to previous results. Second, electron emission intensities follow trends with exposure to $\mathrm{O}_{2}$ and $\mathrm{Cs}$ that mimic previously reported work function behavior. Namely, the effect of oxygen exposure is enhanced for surfaces with larger initial Cs coverage. Note that the data have been scaled slightly to improve readability. The 0.6 ML Cs data were scaled by 0.9 . The 2.4 ML Cs data were scaled by 1.2 . function of the $\mathrm{O}_{2}$ exposure to surfaces with three initial $\mathrm{Cs}$ doses. One can clearly see the influence of oxygen exposure on the emission intensity, which is, furthermore, dependent upon the initial $\mathrm{Cs}$ dose. For a surface with $\sim 0.6 \mathrm{ML} \mathrm{Cs}$ coverage, oxygen exposure results in a barely detectable signal enhancement with a maximum that is between 0.0 and 0.5 Langmuir oxygen exposure. For a surface initially dosed with 1.2 ML Cs, the signal intensity extends to substantially larger oxygen exposures. Fitting this data to an empirical function with the correct asymptotic behavior results in a maximum at 0.5 Langmuir oxygen exposure. For a surface with a $2.4 \mathrm{ML}$ initial Cs dose, a clear maximum in emission is reached around 1 Langmuir of oxygen exposure. These trends are similar to those observed when the work function is monitored versus oxygen exposure for W(110) surfaces with varying initial Cs doses. ${ }^{5,8}$

In control experiments without oxygen exposure carried out in our lab, the electron emission from vibrationally excited NO molecules changed at a much slower rate than that shown in Fig. 5. The rate of change was consistent with that expected from adsorption of background gases, of which $\mathrm{CO}, \mathrm{CO}_{2}$ and $\mathrm{H}_{2} \mathrm{O}$ are the primary constituents. Incidentally, $\mathrm{CO}$ has been shown to be un-reactive on a bulk Cs surface at $90 \mathrm{~K}$, but is easily oxidized by $\mathrm{NO}^{20}{ }^{20}$ We can therefore exclude the influence of background contamination on the results presented in Fig. 5.

The work function of the surfaces prepared here can be approximated by use of photoemission resulting from exposure to the output of a He-Ne laser. Whenever photoemission is observed we can be certain that the work function is below $1.96 \mathrm{eV}(=h v)$. However, the intensity of photoemission is not directly related to the work function. The photoemission intensity is influenced by the photoabsorption characteristics of the surface, mainly through the work function and the probability for electron recapture at defects on the surface. As all of these factors may change during chemical alteration of the surface, the maximum of photoemission may not correspond with the minimum in work function. This has been shown explicitly by Wang. ${ }^{6}$ Our measurements (not shown here) show a similar trend where the photoemission reaches a maximum at slightly smaller exposures than that observed by the electron emission of NO $v=18$. We conclude that the electron emission qualitatively tracks the expected shape of the changing work function.

\section{DISCUSSION}

The major question arising from this work concerns the mechanism for vibrational promotion of electron emission. While we do not attempt to answer this question definitively here, it is interesting to investigate the effect of changing the surface work function on the electron emission induced by collisions of vibrationally excited NO.

The influence of oxygen exposure on exoelectron emission from low work function surfaces has been reported in a number of previous articles. Böttcher et al. investigated the influence of $\mathrm{O}_{2}$ exposure on $\mathrm{Ru}(0001)$ surfaces that had been dosed with $3 \mathrm{ML}$ of Cs. ${ }^{11}$ Similar experiments were carried 
out for $\mathrm{Na}$ on $\mathrm{Ru}(0001) .{ }^{12}$ In these studies, the emission of electrons could not be seen in the limit of zero-oxygen exposure. It was suggested that surface reactions involving a surface oxide were essential to the exoelectron emission mechanism. With $\mathrm{O}_{2}$ on thick Cs, electron emission was explained to be the result of conversion of a transient $\mathrm{A}_{2} \mathrm{O}_{2}$ $(\mathrm{A}=\mathrm{Cs}$ or $\mathrm{Na})$ species into $\mathrm{AO}_{2}$. It was further argued that this reaction proceeds nonadiabatically, resulting in a high energy Auger-like emission event. This chemical mechanism was supported by more detailed studies of Woratschek et al. ${ }^{7}$ There was no obvious correlation between the exoelectron emission and the surface work function.

Analogous work was performed for $\mathrm{NO}$ and $\mathrm{NO}_{2}$ interactions with Cs-dosed $\mathrm{Ru}(0001) .{ }^{21} \mathrm{NO}$ on a $190 \mathrm{~K}$ surface was seen to dissociatively adsorb, without electron emission. After multiple Langmuir NO exposure, exoemission could be seen. When the surface was warmed to room temperature, the exoemission was completely quenched. This is consistent with the observations of Carley, Roberts, and Santra ${ }^{20}$ who showed that NO does not dissociate on Cs surfaces at temperatures exceeding $220 \mathrm{~K}$. At low temperature, $\mathrm{NO}_{2}$ is formed by dissociation first of an $\mathrm{NO}$ molecule and then recombination of the adsorbed $\mathrm{O}$ atom with another $\mathrm{NO}$ molecule. The delayed emission seen in Böttcher et al. ${ }^{21}$ from $\mathrm{NO}$ is attributed to a buildup of $\mathrm{O}$ atoms on the cold surface before reaction with $\mathrm{NO}$ can occur.

In contrast to the just discussed experiments of others, the results seen here are qualitatively different. In particular, two features of the data stand out. As discussed above, the Auger mechanism was observed in systems that showed a delayed onset of electron emission with $\mathrm{O}$ coverage. Here, strong electron emission is observed in the limit of zero-oxygen coverage. In other words, there is no requirement for an oxidized surface for electron emission to occur. Also, electron emission intensities follow a trend which mimics previously reported work function behavior, again exhibiting a significant difference from results seen in other low work function systems.

\section{CONCLUSION}

We observe electron emission due to collisions of highly vibrationally excited NO molecules with a series of low work function surfaces. Systematic measurements of the electron emission signal from NO $v=18$ were carried out as a function of oxygen exposure for surfaces with varying initial Cs coverage. We note a remarkable similarity between trends of the vibrationally produced electron emission as the surface composition changes and reports on surface work function. It appears that the mechanism operative in these experiments is sensitive to the surface work function. While further work is needed to fully characterize the mechanism, these results suggest a direct conversion of vibrational energy into electronic excitation, consistent with a violation of the Born-Oppenheimer approximation.

Nonadiabatic interactions such as the ones discussed in this article have ramifications for a broad range of processes in chemistry and physics which have to do with fundamental processes at the molecular level. A comprehensive understanding of the universality of violations of the BornOppenheimer approximation will enable development of more accurate theoretical models.

\section{ACKNOWLEDGMENTS}

The work was supported by a grant from the National Science Foundation (CHE0135581) and a grant from the Department of Energy Office of Basic Energy Sciences (DEFG02-03ER15441).

${ }^{1}$ M. Born and E. Oppenheimer, Ann. Phys. 84, 457 (1927).

${ }^{2}$ Y. H. Huang, C. T. Rettner, D. J. Auerbach, and A. M. Wodtke, Science 290, 111 (2000).

${ }^{3}$ S. M. Li and H. Guo, J. Chem. Phys. 117, 4499 (2002)

${ }^{4}$ D. R. Lide, Journal of the American Chemical Society (CRC, Boca Raton, FL, 2000), Vol. 122, p. 12614.

${ }^{5}$ W. Maus-Friedrichs, H. Hoermann, and V. Kempter, Surf. Sci. 224, 112 (1989).

${ }^{6}$ C. S. Wang, J. Appl. Phys. 48, 1477 (1977).

${ }^{7}$ B. Woratschek, W. Sesselmann, J. Kuppers, G. Ertl, and H. Haberland, J. Chem. Phys. 86, 2411 (1987).

${ }^{8}$ H. Brenten, H. Mueller, W. Maus-Friedrichs, S. Dieckhoff, and V. Kempter, Surf. Sci. 262, 151 (1992).

${ }^{9}$ T. Greber, Curr. Opin. Solid State Mater. Sci. 3, 446 (1998).

${ }^{10}$ A. Böttcher, R. Imbeck, A. Morgante, and G. Ertl, Phys. Rev. Lett. 65 , 2035 (1990); T. Greber, R. Grobecker, A. Morgante, A. Böttcher, and G. Ertl, ibid. 70, 1331 (1993); A. Böttcher, A. Morgante, T. Giessel, T. Greber, and G. Ertl, Chem. Phys. Lett. 231, 119 (1994); R. Grobecker, H. Shi, H. Bludau, T. Hertel, T. Greber, A. Böttcher, K. Jacobi, and G. Ertl, Phys. Rev. Lett. 72, 578 (1994); A. Böttcher, A. Morgante, and G. Ertl, Surf. Sci. 359, L461 (1996).

${ }^{11}$ A. Böttcher, R. Grobecker, R. Imbeck, A. Morgante, and G. Ertl, J. Chem. Phys. 95, 3756 (1991)

${ }^{12}$ A. Böttcher, R. Grobecker, T. Greber, A. Morgante, and G. Ertl, Surf. Sci. 280, 170 (1993).

${ }^{13}$ J. D. White, J. Chen, D. Matsiev, D. J. Auerbach, and A. M. Wodtke, Nature (London) 433, 503 (2005).

${ }^{14}$ C. Kittrell, E. Abramson, J. L. Kinsey, S. A. McDonald, D. E. Reisner, R. W. Field, and D. H. Katayama, J. Chem. Phys. 75, 2056 (1981).

${ }^{15}$ M. Silva, R. Jongma, R. W. Field, and A. M. Wodtke, Annu. Rev. Phys. Chem. 52, 811 (2001).

${ }^{16}$ J. Chen, D. Matsiev, J. D. White, M. Murphy, and A. M. Wodtke, Chem. Phys. 301, 161 (2004).

${ }^{17}$ Our crystal at one time was Au (111) but we strongly suspect that surface has lost its ordered structure and may have been twinned through use.

${ }^{18}$ M. Skottke-Klein, A. Böttcher, R. Imbeck, S. Kennou, A. Morgante, and G. Ertl, Thin Solid Films 203, 131 (1991).

${ }^{19}$ C. Amiot, J. Mol. Spectrosc. 94, 150 (1982); R. Engleman, R. E. Rouse, H. M. Peek, and V. D. Baiamonte, Report No. LA-4364, 1970.

${ }^{20}$ A. F. Carley, M. W. Roberts, and A. K. Santra, Surf. Sci. 516, L523 (2002).

${ }^{21}$ A. Böttcher, R. Grobecker, T. Greber, and G. Ertl, Chem. Phys. Lett. 208, 404 (1993). 